\title{
Germinoma with synchronous involvement of the pineal gland and the suprasellar region: a treatable cause of visual failure in a young adult
}

\begin{abstract}
Germinomas constitute $0.1-3.4 \%$ of all intracranial tumours ${ }^{1}$ and $90 \%$ affect patients less than 20 years old. Although they are relatively aggressive tumours, which commonly invade the surrounding tissue and seed within the central nervous system, they are very radiosensitive and carry a favourable prognosis. We describe a 19-year-old man who presented with bilateral visual failure secondary to a germinoma with synchronous involvement of the pineal gland and the suprasellar region. Radiotherapy restored his vision almost back to normal.

Eye (2004) 18, 525-526. doi:10.1038/sj.eye.6700715

Keywords: germinoma; pineal gland; visnal loss
\end{abstract}

\section{Case report}

A 19-year-old man presented with a 5-month history of progressive bilateral visual impairment. He did not complain of any other symptoms. There was no family history of visual failure and no history of drug abuse or exposure to toxins. Visual acuity was counting fingers on the right and 6/24 on the left. There was a right afferent pupillary defect. Eye movements were normal. Dilated fundus examination revealed bilateral pale optic disks but normal macula and retina. Slit lamp examination was normal. Intraocular pressures were within the normal range. General clinical examination was unremarkable and there were no other abnormal neurological signs. An MRI
S Hadjikoutis and T Hughes scan of the brain with gadolinium showed abnormal contrast enhancement of the suprasellar region and pineal gland (Figure 1). The radiological findings were consistent with intracranial germinoma. An MRI scan of the spinal cord was normal, and ultrasound of the scrotum and abdomen were unremarkable. His routine bloods and chest $\mathrm{X}$-ray were normal. Serum angiotensin-converting enzyme (ACE) was normal, and autoantibody screen was negative. His alpha-fetoprotein (AFP) and human chorionic gonadotrophin (HCG) in the blood and cerebrospinal fluid (CSF) were normal. His CSF was acellular with normal opening pressure, protein and glucose. Visual evoked potentials were reduced in amplitude, but not delayed, that is, compatible with bilateral optic neuropathy. An electroretinogram was normal.

He received cranio-spinal radiotherapy to a dose of $30 \mathrm{~Gy}$ in 20 fractions, with a further $24 \mathrm{~Gy}$ in 15 fractions to the tumour bed. His visual acuity improved gradually and 12 months later was $6 / 6$ (right) and 6/9 (left). Two repeated MRI scans -2 and 8 months after radiotherapy - of the brain and spinal cord with gadolinium were normal. There has been no recurrence of his symptoms after 15 months follow-up.

\section{Comment}

Germinomas arise because of neoplastic changes of germ cells during embryonic development. They are the most common intracranial germ cell tumours and are
Department of Neurology University Hospital of Wales Cardiff, UK

Correspondence: $S$ Hadjikoutis Department of Neurology University Hospital of Wales Heath Park Cardiff CF23 6HQ, UK

E-mail: savvashadjikoutis@ yahoo.com

Received: 5 April 2003 Accepted in revised form: 2 July 2003 
a

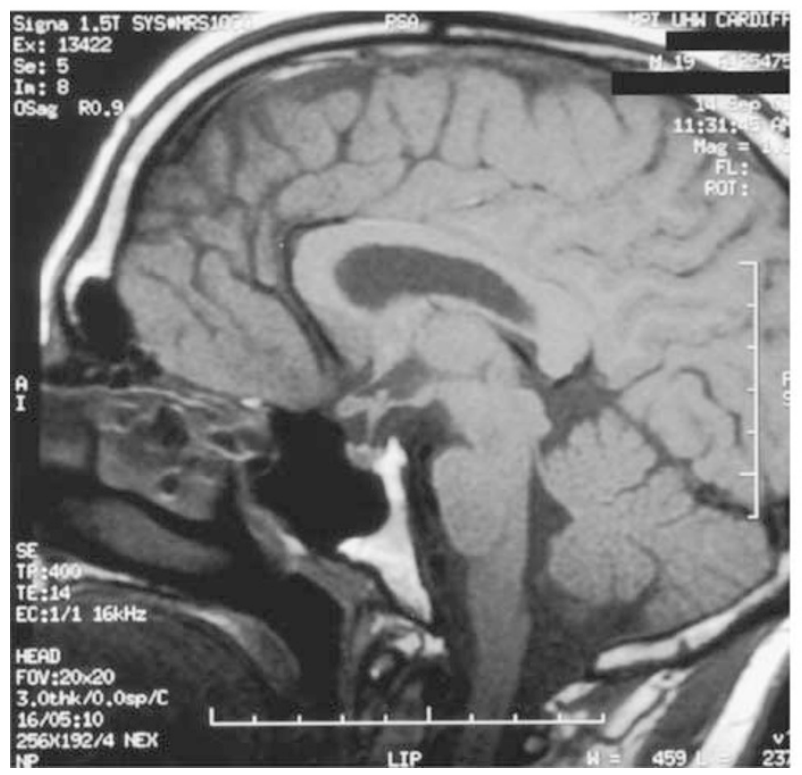

b

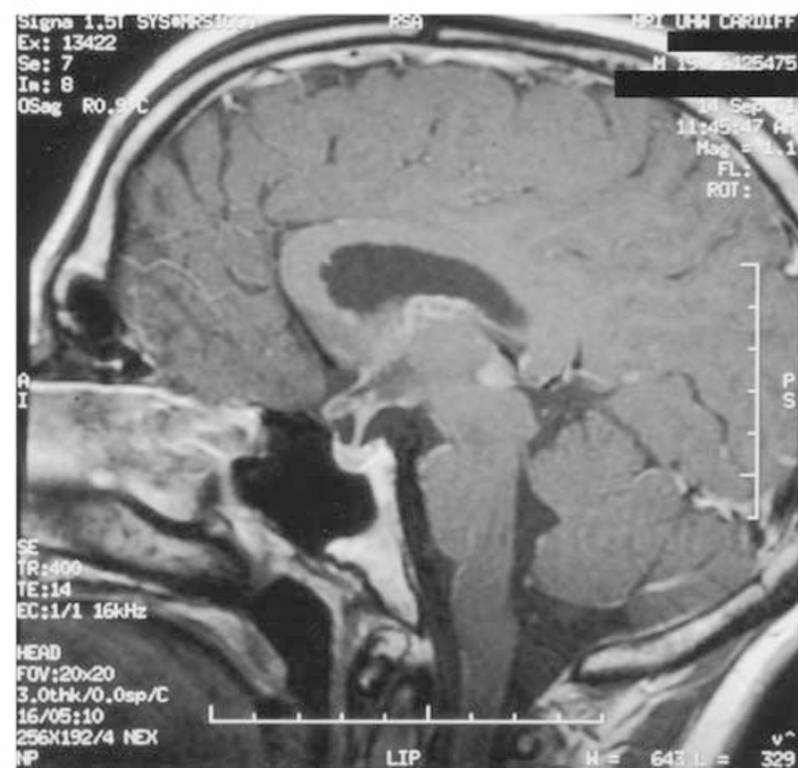

Figure 1 Sagittal pre (a) and post (b) contrast T1-weighted MRI brain images showing extensive abnormal contrast enhancement passing along the suprasellar region and pineal gland.

histologically indistinct from testicular seminoma. Most germinomas arise in the midline axis from the supraseller cistern to the pineal region, ${ }^{2}$, and visual failure is a common presentation. The mechanism involved is presumably infiltrative. They usually affect young patients ( $<20$ years) with peak age at $10-12$ years. Men are more likely to develop a geminoma (male/ female $4: 1$ ). MRI scan of the brain with gadolinium is the recommended imaging. ${ }^{3}$ Germinoma is usually so small that it is hard to see on unenhanced MRI, but it brightens prominently and homogeneously with contrast. Multiple lesions are seen in almost $50 \%$ of cases.

The role of surgery, beyond biopsy for tissue diagnosis, remains controversial. Surgical resection exposes the patient to the operative risks and the possibility of regional tumour dissemination. Germinomas are exquisitely sensitive to radiotherapy. The presence of a relatively homogeneous, well-circumscribed, extra-axial, enhancing pineal region mass in a young male is so characteristic of a germinoma that diagnostic radiotherapy can be justified. ${ }^{4}$ Germinomas characteristically infiltrate locally, and spread by dissemination through the craniospinal and subarachnoid spaces. MRI scan of the entire neuraxis is necessary to search for CSF dissemination. HCG is only occasionally elevated with central nervous system germinomas, while the level of AFP is rarely elevated. Elevated levels of HCG and AFP are more closely correlated with malignant germ cell tumours such as embryonal carcinoma, endodermal sinus tumours, and choriocarcinoma. Spinal irradiation is recommended to cover for possible CSF invasion. As a consequence of improving the means of diagnosis and radiotherapy, the current survival rate of patients with germinoma is $90 \%$ after a median follow-up of 5 years, and a 20-year survival rate of $80 \%$ has been reported. ${ }^{5}$

Intracranial germinomas frequently involve the visual pathways and may thus present with visual failure in the young adult and paediatric population. They are rewarding tumours to identify, because, if properly diagnosed and managed, they can be eradicated or controlled.

\section{References}

1 Jennings MT, Gelman R, Hochberg F. Intracranial germ-cell tumours: natural history and pathogenesis. J Neurosurg 1985; 63: 155-167.

2 Horowitz MB, Hall WA. Central nervous system germinomas. Arch Neurol 1991; 48: 653-657.

3 Liang L, Korogi Y, Sagahara T. MRI of intracranial germ-cell tumours. Neuroradiology 2002; 44: 382-388.

4 Jakacki R. Central nervous system germ-cell tumours. Curr Treat Options Neurol 2002; 4: 139-145.

5 Hudgins RJ, Hudgins PA. Pineal region tumours in children. Contemp Neurosurg 1990; 12: 1-6. 